\title{
Supervised Learning in the Brain
}

\author{
Eric I. Knudsen \\ Department of Neurobiology, Stanford University School of Medicine, Stanford, California 94305-5401
}

Experience shapes the functional organization of the brain, optimizing and customizing its properties for the individual and his or her environment. One way that experience shapes the constituent networks of the brain is through supervised learning. In supervised learning, information from one network of neurons acts as an instructive signal to influence the pattern of connectivity in another network. As a result, the instructed network learns to process information so that a particular goal or transformation specified by the instructive signal is achieved. In so doing, supervised learning establishes patterns of connectivity efficiently and with a precision that does not need to be and, often, cannot be encoded in the genome.

Supervised learning contributes to the development and maintenance of a variety of brain functions. For example, sensorimotor networks that control goal-directed movements are calibrated by sensory feedback indicating the accuracy with which the movements are made. In a specific example that will be discussed at some length, a visual instructive signal, indicating the slip of images across the retinae, is used to calibrate the transformation of vestibular sensory information (indicating rotation of the head) into precise, compensatory movements of the eyes that stabilize the images on the retinae (Miles and Eighmy, 1980). Supervised learning can also control the representation of information in sensory networks. For example, in the development of binocular neurons in the optic tectum of the frog Xenopus, visually driven activity from the contralatcral eye specifies the topography of the visual map originating from the ipsilateral eye (Gaze et al., 1970; Udin, 1985). In this example, which also will be discussed in detail, the activity from the contralateral eye provides an instructive signal that assures the mutual alignment of left- and right-eye receptive fields. It is likely that supervised learning also contributes to the establishment of networks that support certain cognitive skills, such as pattern recognition and language acquisition, although there is, as yet, no experimental confirmation of this proposition.

This article discusses supervised learning as it might be implemented in the brain. Different kinds of instructive signals,

\footnotetext{
Key words: long-term potentiation, experience-dependent plasticity, vestibulo-ocular reflex, classical conditioning, auditory plasticity, cerebellum, optic tectum, superior colliculus
}

I thank Michael Brainard, Steve Esterly, Dan Feldman, Josh Gold, Richard Thompson, and Sue Udin for critical readings of the manuscript. The work was supported by a grant from the National Institute of Deafness and other Communicative Disorders (R01 DC00155-14).

Correspondence should be addressed to Eric I. Knudsen, Ph.D., Department of Neurobiology, Stanford University School of Medicine, Stanford, CA 94305 5401 .

Copyright (C) 1994 Society for Neuroscience $0270-6474 / 94 / 143985-13 \$ 05.00 / 0$ the types of learning rules that could make use of such signals, and neural mechanisms that could mediate such learning are described. These issues are illustrated with biological examples of networks in which the connectivity and neural components involved in supervised learning are known. Finally, unique roles that this form of learning can play in shaping the functional properties of the brain are discussed.

\section{Neural implementation of supervised learning}

The implementation of supervised learning requires a network of neurons that can change its pattern of connectivity (topology and/or strengths of connections), and an instructive signal that can regulate the changes (Fig. 1). In the simplest case, these functions can be subserved by three groups of neurons: input, output, and instructor neurons. For example, in the case of binocular alignment in the optic tectum of Xenopus, the input neurons are the neurons conveying information from the ipsilateral eye, the output neurons are the tectal neurons, and the instructor ncurons are the ncurons conveying information from the contralateral eye (Udin, 1985). The connections between the ipsilateral eye (input) neurons and the tectal (output) neurons are modified depending on the degree to which the activity of the ipsilateral eye neurons agrees with contralateral eye (instructor) activity. The instructive signal is the pattern of activity from the contralateral eye, and the sites of change (learning) are the connections between ipsilateral eye neurons (inputs) and tectal neurons (outputs).

In more general cases, such as the control of goal-directed movement or classical conditioning, the network is an extensive system of interconnected groups of neurons located in many regions of the brain. The input in these cases is provided by a wide variety of sensory or cognitive inputs, the output is represented by movement, and the instructive signal is provided by sensory information about whether the network has translated the input into appropriate behavior.

In the nervous systems studied to date, instructive signals are of two types, activity templates or error signals (Fig. 2). Activity templates dictate to the network how information will be represented: a referent pattern of activity from one network causes the instructed network to process its inputs so that its outputs approximate the activity template. This is exemplified by the activity template established by the retinotectal projection from the contralateral eye that dictates the pattern of the indirect projection from the ipsilateral eye in the tectum of Xenopus (Udin and Keating, 1981). Error signals, on the other hand, indicate whether the transformation carried out by a network was successful in achieving a particular output (Fig. 2). This involves a comparison of the network's output or the consequences of that output (i.e., performance) with information about the goal (which could also be a referent pattern of activity from 


\section{Instructive Signal}

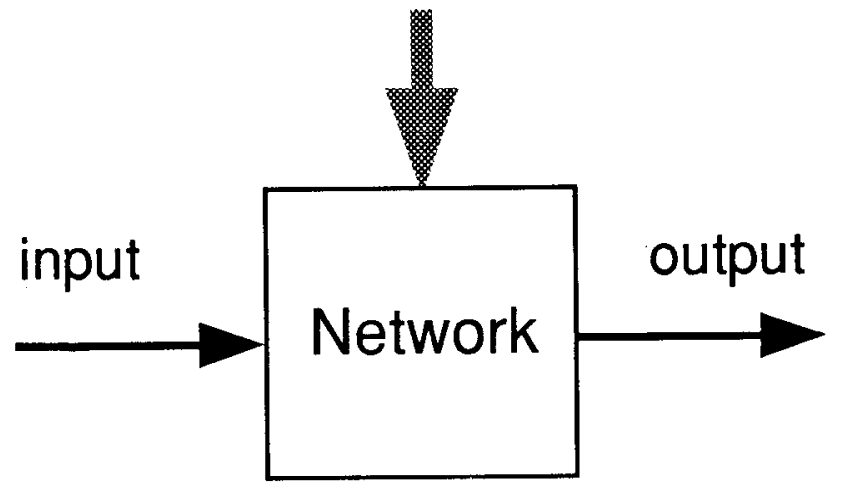

Figure 1. Essential components for supervised learning. The network represents the strength and topology of neuronal connections between the input and the output. The connectivity of the network is modifiable, and the modifications are controlled by an instructive signal.

another network), and a feedback signal indicating the result of this comparison. For example, in the case of reaching movements, the direction of reach is compared with visual information about the location of the target of reach, and errors are fed back into the sensorimotor network to adjust the accuracy of subsequent reaching movements (Marr, 1969; Georgopoulos, 1986).

\section{Rules and mechanisms of learning}

An instructive signal could control the modification of a network in one of several ways. It could simply destabilize the current pattern of connectivity in the modifiable network and enable the network to settle on a new pattern. In this case, the instructional signal indicates when the result of the information processing carried out by the current connectivity pattern is not good enough, but does not provide information about the change that is required to improve the network's performance. It simply signals "try again." This may be how contralateral eye inputs cause indirect ipsilateral eye inputs to find correct postsynaptic targets in the optic tectum of Xenopus (Udin, 1989). Alternatively, the instructive signal could act as a global reinforcement signal, indicating the direction of change in the connectivity pattern that would improve the performance of the network. In this case, the instructive signal might gradually increase the strength of connections when they contribute to appropriate outputs, decrease the strength of connections when they contribute to inappropriate outputs, or do both. Finally, the instructive signal could specify both the direction and magnitude of change that would optimize the performance of the network. This type of signal is used widely in certain classes of mathematical models, such as in backpropagation, to effect learning in computer networks (Anderson and Rosenfeld, 1988; Anderson et al., 1990). Such a signal could change the pattern of neuronal connectivity discretely to provide the output required by the instructor. In each of these ways, an instructive signal could eventually establish a strong, functional connection between neurons that are only weakly coupled before learning.

The mechanisms by which instructive signals alter connections in modifiable networks of neurons are unknown. Mechanisms of synaptic plasticity have been discovered, however, that can support other forms of learning (Lomo, 1966; Ito, 1989;
Mulkey and Malenka, 1992). These mechanisms can account for the capacity of the brain to self-organize during development and to reduce the complexity of encoded information to the dimensions or parameters that are important to the animal (Anderson and Rosenfeld, 1988; Miller, 1989; Anderson et al., 1990; Brown et al., 1991). These kinds of modifications do not require supervision. Although they will not be discussed here, the rules and mechanisms that underlie them can be the same as those that underlie supervised learning.

The rules that appear to support self-organization and dimension reduction in the brain are based on the correlation of activity in pre- and postsynaptic neurons. One of these rules is the modern variant of the Hebbian rule: synapses whose activity consistently correlates with strong depolarization in the postsynaptic neuron are strengthened, while those whose activity does not are weakened (Hebb, 1949; Stent, 1973; Brown et al., 1991). The kinds of mechanisms that may support this form of synaptic strengthening and weakening are those of long-term potentiation (LTP) and long-term depression (LTD), respectively, as have been found in the hippocampus (Lomo, 1966; Bliss and Lomo, 1973; Mulkey and Malenka, 1992) and some regions of neocortex (Bindman et al., 1988; Brown et al., 1988; Kirkwood et al., 1993). A second learning rule is the anti-Hebbian rule: synapses whose activity correlates with strong depolarization of the postsynaptic neuron are weakened. A mechanism that supports the weakening of synapses based on strong, correlated activity is referred to as anti-Hebbian LTD, as has been found in slice preparations from the cerebellum and striatum (Hirano and Hagiwara, 1988; Ito, 1989; Calabresi et al., 1992).

It is possiblc that in the cases in which Hebbian or antiHebbian mechanisms are at work, the fundamental difference between supervised and unsupervised learning is that in unsupervised learning, the connectional strengths of all inputs are equally modifiable, and the strength of any given input connection can be weakened to the point where it no longer contributes to activity in the postsynaptic neuron. In contrast, in supervised learning, the instructive inputs are dominant in that their activity either depolarizes or hyperpolarizes postsynaptic neurons powerfully, and their connectional strengths are unaffected by the activity that occurs in the postsynaptic neurons. Under these conditions, only the modifiable inputs that are active concurrently with the instructive inputs are strengthened, in the case of LTP, or weakened, in the case of anti-Hebbian LTD. Thus, the activity of the instructive input will eventually determine the transformation carried out by the postsynaptic neuron on the information contained in the activity of its inputs. This transformation is "learned" in the sense that, once established, it will be carried out by the network in the absence of instructive input activity.

Results from a variety of nervous systems support the hypothesis that the mechanisms underlying Hebbian and antiHebbian learning may also underlie supervised learning. The following is a summary of these results for four of the more thoroughly understood networks that exhibit supervised learning, namely, networks involved in classical conditioning, the vestibulo-ocular reflex, binocular receptive field alignment, and the creation of an auditory map of space.

\section{Cerebellar networks involved in classical conditioning}

A discrete response that is triggered by a stimulus (unconditioned response, UR) can eventually be evoked by an unrelated 


\section{ACTIVITY TEMPLATE}

\section{Before Learning}

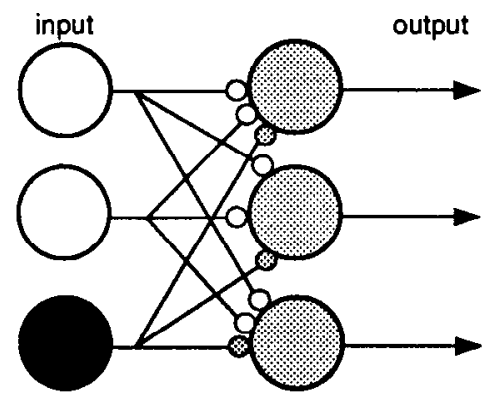

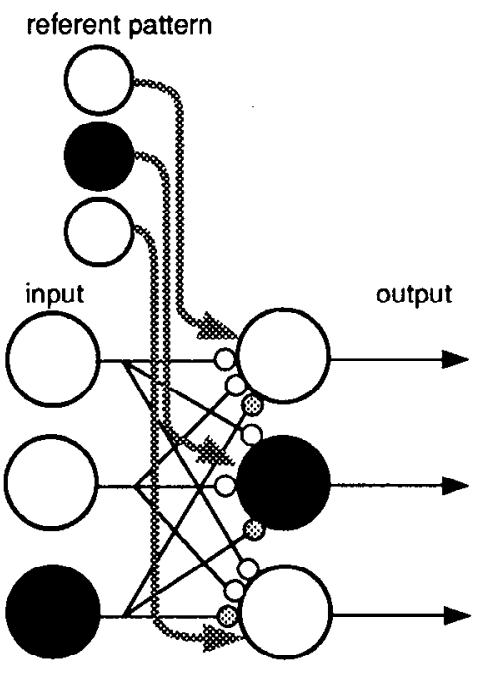

ERROR SIGNAL
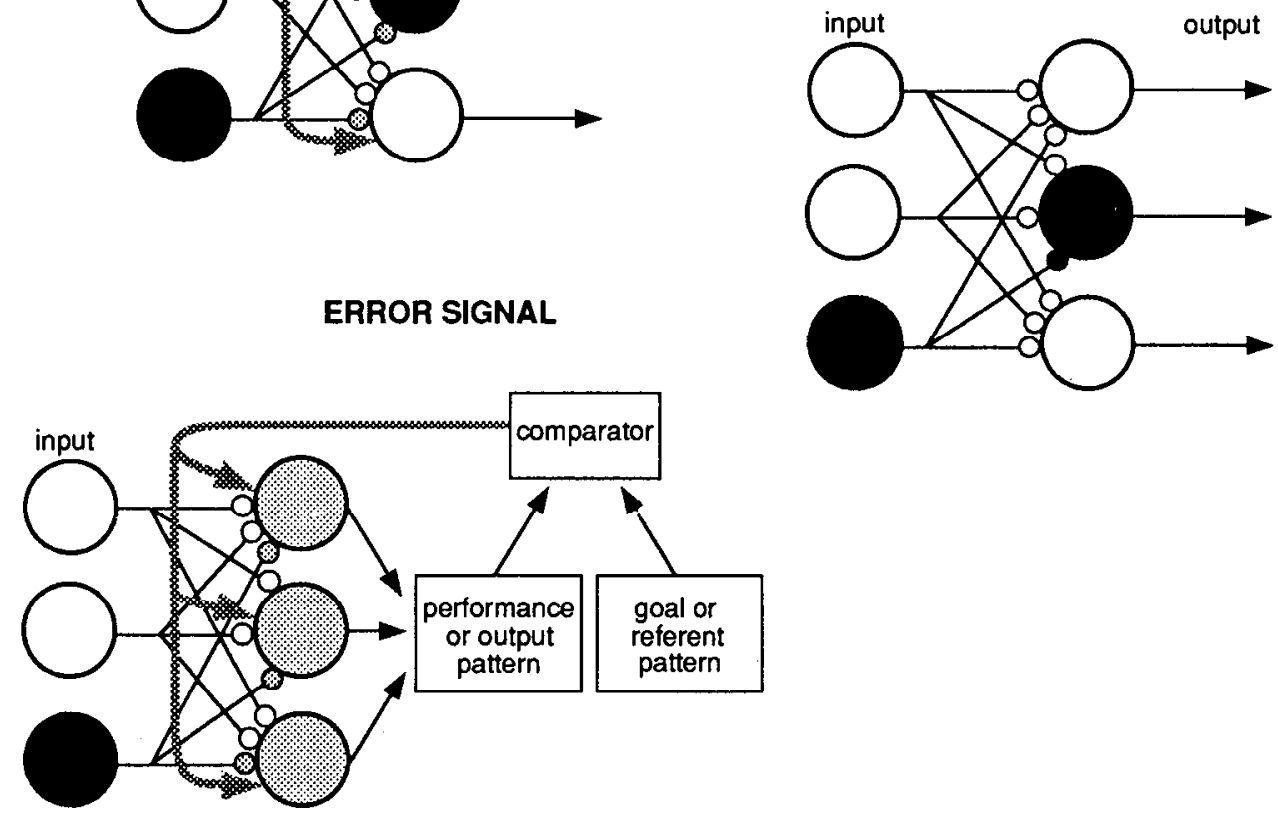

Figure 2. Instruction of a modifiable network by an activity template or an error signal. Each large circle represents a neuron, and its shading represents its stimulus-driven activity level. Connections between input and output neurons are represented by small circles. The shading of connections indicates the product of activity level and connectional strength. The network is shown on the left before learning takes place. All connections are of equal strength, and a high activity level in the lower input neuron (black) results in an intermediate activity level (gray) in all output neurons. After learning (shown on the right), high activity in the same input neuron results in a high activity level in only the middle output neuron, because of the strengthening of the connection between the lower input and the middle output neuron and the weakening of the connections between the lower input ncuron and the upper and lower output neurons. This change in connectivity may result from the instructive influence of an activity template (upper panel) or from an error signal (lower panel). The activity template (referent pattern) originates in another network and is imposed by dominating connections (thick arrows) on the modifiable network. If the learning rule is Hebbian, then connections will be strengthened between concurrently active input and output neurons, where the activity of the output neurons is driven by the activity of the referent pattern. Conversely, the connections between input and output neurons will be weakened between neurons that are not active concurrently. The result of learning is that this particular input pattern will eventually elicit the same pattern of activity in the output neurons that is imposed by the referent pattern. If the learning rule is anti-Hebbian (not shown), then the learned output pattern would be the complement of the referent pattern. The error signal results from a comparison of the performance or output pattern of a network with the goal or desired output pattern (e.g., the referent pattern shown above). The error signal feeds back on the modifiable network via dominating connections (thick arrows). The comparator could provide a single distributed error signal that reflects the overall quality of match between performance and goal. When the error signal indicates that the match is good, recently active connections would be strengthened, and when the match is poor, recently active connections would be weakened. Alternatively, the comparator could provide an error signal that specifies the direction and, possibly, the magnitude of the changes in connectional strength required to produce a better match between performance and goal.

stimulus, if the unrelated stimulus (conditioned stimulus, CS) consistently predicts the occurrence of the trigger stimulus (unconditioned stimulus, US). For example, an animal will learn to blink an eye in response to a tone (CS), if the tone consistently predicts the occurrence of an airpuff delivered to the cornea of the eye (US). The eyeblink thus becomes a conditioned response (CR). This process can be represented as follows: corneal airpuff (US) $\rightarrow$ eyeblink (UR) tone (CS) + corneal airpuff (US) $\rightarrow$ eyeblink (UR)

tone (CS) $\rightarrow$ eyeblink (CR)

This form of learning is referred to as classical conditioning (Pavlov, 1927; Black and Prokasy, 1972; Thompson, 1986). 


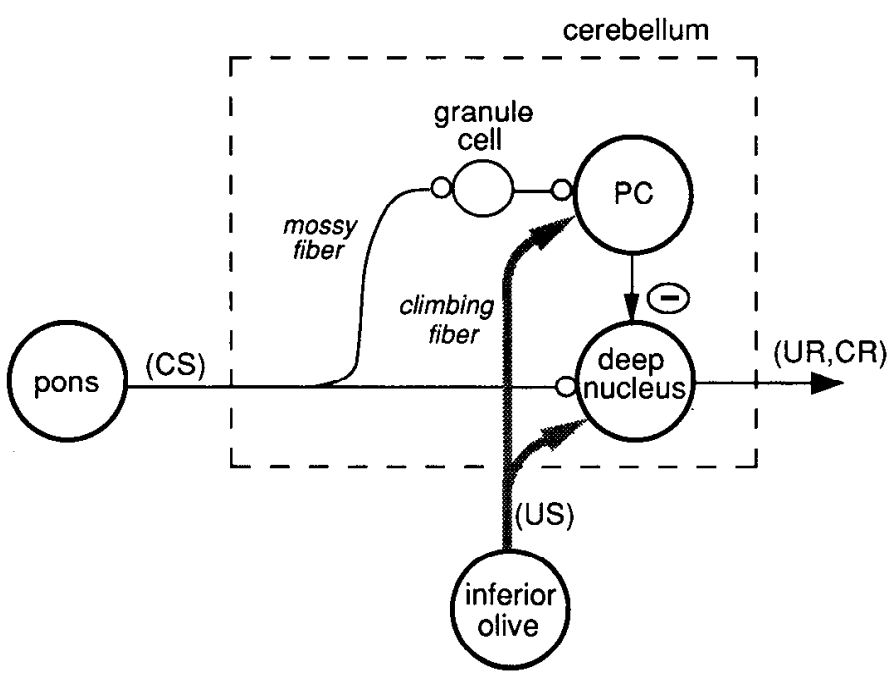

Figure 3. Neural pathways in the cerebellum involved in supervised learning during classical conditioning. Large circles represent particular populations of neurons. The neurons that reside in the cerebellum are enclosed by the dashed box. Inputs enter and outputs leave the cerebellum via the cerebellar peduncles. Neurons in the inferior olive provide an instructive signal (thick arrows) to Purkinje cells $(P C)$ and to neurons in the deep nuclei, the proposed sites of plasticity. Output from the deep nuclei is conveyed to several target nuclei, a major target being the red nucleus. Purkinje cells inhibit (minus sign) deep nuclei neurons; all other connections are excitatory. $C S$, conditioned stimulus; $C R$, conditioned response; $U S$, unconditioned stimulus; $U R$, unconditioned response.

Classical conditioning involves the formation of a strong, functional connection between input neurons that are activated by the CS and output neurons that can mediate the IJR. The instructive signal that supervises the learning is the input activity associated with the US. The CS network that becomes functionally connected to the UR network is selected out of the sensory repertoire of the animal based on the temporal pairing of the CS input with the US input.

One site of supervised learning during classical conditioning appears to be in the cerebellum. Eyeblink conditioning in rabbits is prevented if paired tone and corneal airpuff stimuli are presented while neurons in the cerebellum (Fig. 3) are inhibited with a local injection of the GABA agonist muscimol (Krupa et al., 1993). This result indicates that neurons in the cerebellar cortex or deep cerebellar nuclei are either the site of learning, or that they provide an essential input to the site of learning. In contrast, if paired tones and corneal airpuffs are presented while neurons in the red nucleus (a primary target of deep nucleus neurons) are inhibited, classical conditioning does occur, even though the CR is not expressed while the red nucleus remains inhibited. This result indicates that the learning occurs before activity reaches the red nucleus. These results provide strong evidence for a site of learning in the deep nuclei and/or cortex of the cerebellum.

Neurons that carry a signal associated with the CS to cells in the deep nuclei and the cortex are located in the pontine nuclei (Fig. 3). These neurons, which receive sensory information from ascending and descending sensory pathways, send axons by way of the middle cerebellar peduncle to neurons in the deep nuclei and highly branching "mossy fiber" projections to cortical granule cells which, in turn, excite cortical Purkinje cells. Accordingly, lesions of the middle cerebellar peduncle abolish CRs that are already learned and prevent the acquisition of new CRs, presumably because activity evoked by the CS no longer reaches the cerebellum (Solomon et al., 1986). Moreover, repeated pairing of electrical microstimulation of the middle cerebellar peduncle with a US causes strong CRs to develop in response to electrical microstimulation of the peduncle alone (Steinmetz et al., 1986).

Neurons that carry US activity are located in the dorsal accessory and inferior olive (Fig. 3). The axons of these neurons enter the cerebellum through the inferior cerebellar peduncle These fibers send collaterals to neurons in the deep nuclei as well as "climbing fiber" projections to Purkinje cells in the cortex. Electrical microstimulation of the inferior olive evokes a discrete movement and can substitute for a naturally delivered US (Mauk et al., 1986). If microstimulation in the olive is consistently preceded by a CS, the movement that is evoked becomes a CR. In fact, if electrical microstimulation in both the inferior olive and the mossy fiber projection is repeatedly paired, mossy fiber stimulation alone will eventually evoke whatever discrete movement (UR) was elicited previously by inferior olive stimulation alone (Steinmetz et al., 1989). In addition, lesions in the olive can prevent new CRs from being learned and cause learned CRs to disappear gradually (be "extinguished") with continued training (McCormick et al., 1985), as if the instructive signal were removed from the site of learning.

CS and US inputs converge on cells in the deep nuclei and on Purkinje cells in the cortex (Fig. 3), and a neurophysiological correlate of classical conditioning is observed in the activity of these two cell types (McCormick and Thompson, 1984). Before training, deep nuclei and Purkinje cells may respond either to the CS or the US, or they may discharge in association with the UR. Over the course of training, new patterns of activity develop that are elicited by the CS and that precede and predict the CR. In the cortex, these consist of increases or decreases of Purkinje cell activity in response to the CS that last through the CR. In the deep nuclei, they consist of increases in discharge frequency that reflect the magnitude and time course of the CR (Fig. 4). It is likely, therefore, that supervised learning takes place at the level of Purkinje and deep nuclei cells where modifiable inputs and instructive signals converge.

The dominating synapse that is proposed to convey the instructive signal, according to the model for supervised learning, is present in the cerebellar cortex. A climbing fiber contacts a single Purkinje cell at multiple sites along its primary and secondary dendrites. Due to the strength of this connection, whenever the climbing fiber discharges, the Purkinje cell depolarizes strongly and discharges an intense burst of action potentials (Llinás and Hillman, 1969). As originally proposed by Marr (1969), the instructive activity carried by climbing fibers could therefore control the connectional strength of granule cell inputs to Purkinje cells.

The signal carried by climbing fibers can be viewed as an activity template in this system (Fig. 2). The observation that learning proceeds even when the red nucleus is inactivated and no CRs are expressed (Krupa et al., 1993) demonstrates that no error signal based on the behavior is needed for learning to occur. Instead, the powerful climbing fiber activity evoked by the US, acting as a template, could cause the connectional strengths of any sensory inputs that are consistently correlated with it to increase. After sufficient repetition, the activity of these sensory inputs alone would drive the UR pathway.

Alternatively, the signal carried by climbing fibers may rep- 
resent an error signal (Fig. 2) that is generated independently of behavior: climbing fiber activity could represent the difference between the existing activity pattern in the cerebellar network and the goal activity pattern, that is, one that normally would evoke a CR. Consistent with this view, climbing fiber activity elicited by a US is strong before training, and decreases dramatically as a CR is acquired, as would be expected of an error signal (Thompson, 1990).

An LTP-like mechanism, operating in the cortex or deep nuclei, could readily explain the associative learning that is manifest in the responses of these cells during classical conditioning (Fig. 4). Despite much effort, however, evidence for LTP has not been found in the cerebellum. Instead, the only mechanism of associative synaptic plasticity that has been demonstrated convincingly is anti-Hebbian LTD in culture and slice preparations of the cerebellar cortex (Ito, 1984, 1989; Hirano and Hagiwara, 1988). Repetitive coactivation of climbing fiber and granule cell (mossy fiber) inputs in these preparations results in a weakening of granule cell synapses onto Purkinje cells. Even if this anti-Hebbian LTD does occur also in vivo, however, it is not clear how this particular mechanism could explain the observed increases or decreases in Purkinje cell activity that are evoked by excitatory CS input after conditioning (McCormick and Thompson, 1984; Thompson, 1990).

The constituents of supervised learning - cell types involved, connections, and electrophysiological properties - have been described in greatest detail in this system. Critical aspects of the system are still unresolved, however. Foremost among these are direct demonstrations of appropriate synaptic plasticity in the cortex or in the deep cerebellar nuclei that result from classical conditioning, and the elucidation of mechanisms of synaptic plasticity operating in vivo that could underlie the learning.

\section{Brainstem networks involved in the vestibulo-ocular reflex}

Another problem that the brain solves with supervised learning is the calibration of movements. The vestibulo-ocular reflex, or VOR, is a model system for the adaptive control of movement. The goal of the VOR is to stabilize the visual images on the retinae during head rotations by rotating the eyes at an equivalent speed in the opposite direction. If the VOR is inaccurately calibrated, the visual images slip across the retinae and vision is severely degraded.

The "gain" of the VOR is quantified as the angular speed of eye rotation divided by the angular speed of head rotation, a ratio that ideally should equal unity. A change in VOR gain (Fig. $5 A$ ) can be induced experimentally by presenting an animal with a full-field visual stimulus that moves during rotations of the head (Watanabe, 1984). If the stimulus is moved consistently in the same direction as the head rotation, the reflexive counterrotation of the eyes will initially be too great to stabilize the stimulus on the retinae and the gain of the VOR will gradually decrease. Conversely, if the visual stimulus is moved consistently in the opposite direction, the gain of the VOR will gradually increase. The instructive signal results from the slip of the visual images across the retinae (retinal slip), which the VOR is designed to minimize.

A portion of the network that contributes to the regulation of VOR gain is shown in Figure $5 B$. When the head rotates, vestibular afferents encode information about the angular velocity of rotation as an increase or decrease (depending on the direction of rotation) of tonic excitatory activity. This vestibular activity is transmitted to Purkinje cells in the floccular lobe of

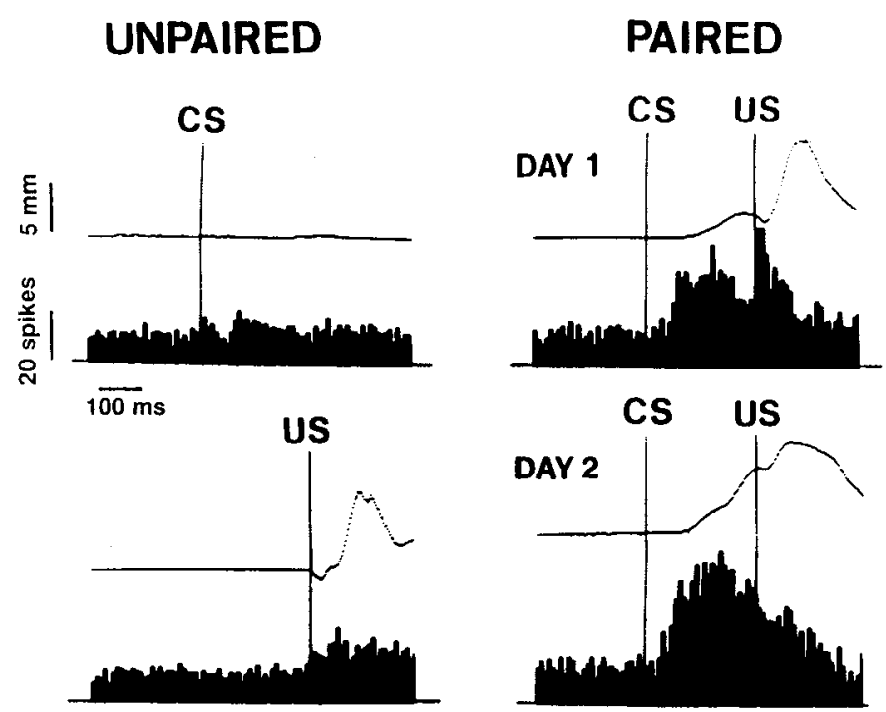

Figure 4. Neuronal response properties in the deep cerebellar nuclei of a rabbit before and after classical conditioning. The conditioning paradigm involved pairing a tone $(C S)$ with a corneal airpuff $(U S)$ to elicit an eyeblink $(U R)$. The upper trace in each panel represents the movement of the nictitating membrane, with up being extension of the membrane across the eyeball. Extracellular unit responses are shown as histograms in which the height of a bar indicates the number of spikes occurring within a $9 \mathrm{msec}$ interval. As shown on the left, the CS elicited little, if any, response before training (unpaired). During the first day of training (day 1), both the nictitating membrane and the units began responding to the CS (movement and unit activity occurring before the US). As training continued and learning progressed (day 2), unit responses to the CS increased. Data from McCormick and Thompson (1984).

the cerebellum via mossy fiber projections to granule cells. Information about eye velocity and retinal slip is also conveyed to these Purkinje cells via mossy fiber projections (Lisberger, 1988). The vestibular activity is sent in parallel to a subset of neurons in the medial vestibular nucleus that receives tonic inhibitory input from the floccular Purkinje cells. Because these medial vestibular neurons receive an input from the floccular lobe, they are referred to as floccular target (FT) neurons (Lisberger and Pavelko, 1988). FT neurons, in turn, convey the information about head rotation to oculomotor neurons that control the movements of the eyes.

During the execution of a normal VOR, the tonic activity conveyed by the vestibular and eye velocity inputs to floccular Purkinje cells changes in a complementary ashion so that Purkinje cell discharge rates remain essentially constant. When the gain of the VOR is incorrect, however, a retinal slip signal modulates the activity of the Purkinje cells via the mossy fiber pathway and via a climbing fiber pathway from the inferior olive (Watanabe, 1984). The climbing fiber pathway also sends collaterals, and presumably the retinal slip signal, to FT neurons in the medial vestibular nucleus (Balaban, 1984). The retinal slip signal causes the network to adjust the gain of the VOR.

Vestibular inputs do not change their sensitivity to head rotations with changes in VOR gain (Lisberger and Miles, 1980; Miles and Braitman, 1980). In contrast, FT neurons do (Lisberger and Pavelko, 1988): following an experimentally induced increase in VOR gain, for example, FT neurons that drive the eyes in the direction opposite to that of the head exhibit a marked increase in their response to a standard head rotation. This indicates that adaptive synaptic plasticity resulting from 
A

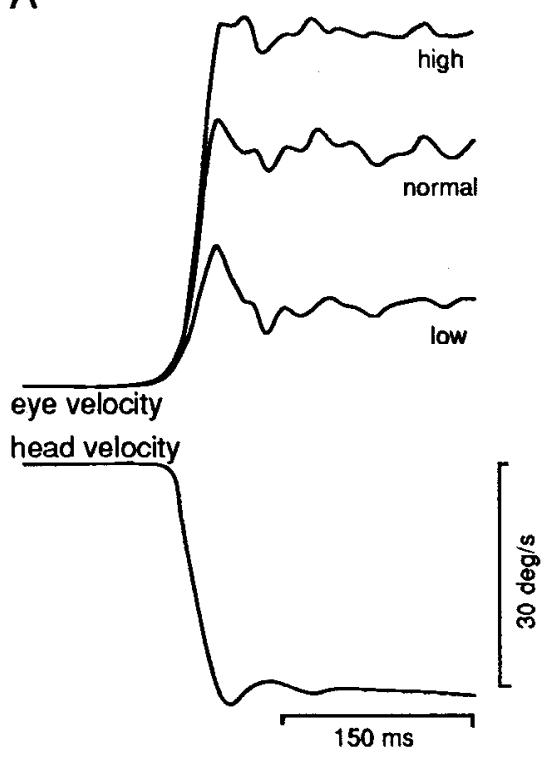

B

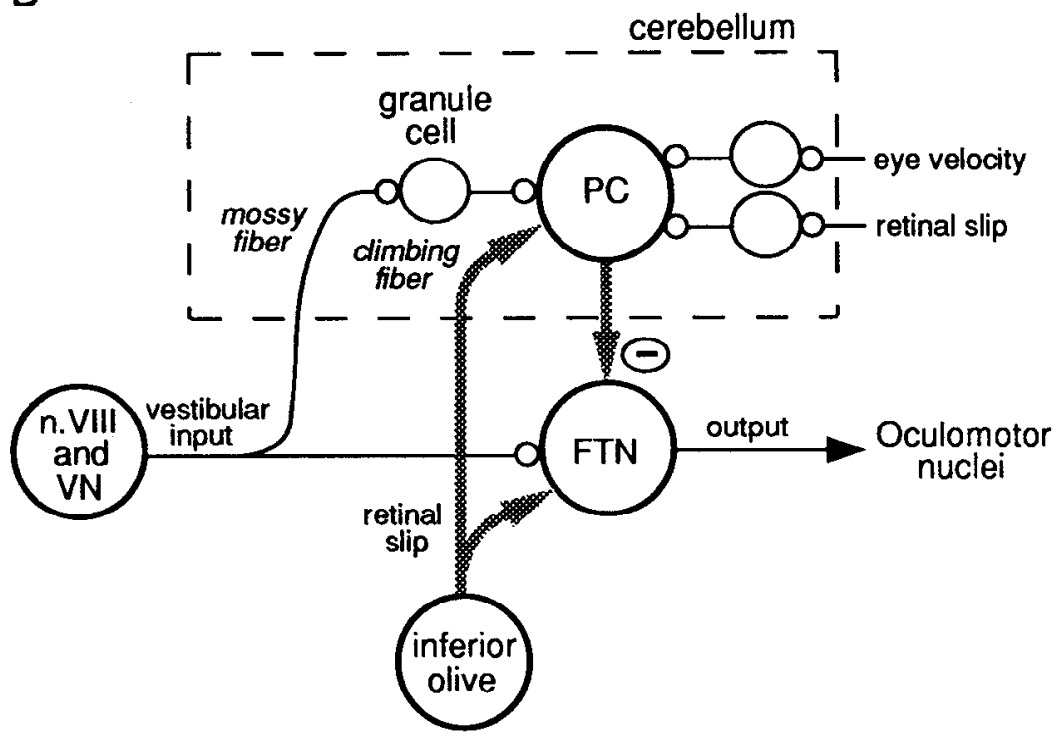

Figure 5. Learning exhibited by the VOR and the proposed sites of plasticity in the monkey. In $A$, search coil recordings of eye movements (upper three traces) are shown in response to a transient rotation of the head (lower trace) with the VOR in a low, normal, or high gain state. Changes in VOR gain were induced by magnifying (high) or miniaturizing (low) spectacles worn during forced head rotations. Each trace is the average of 10 traces of eye or head velocity. Upward deflections are rightward motion. Data from Lisberger (1988). In $B$, a portion of the neural pathway involved in the supervised learning is shown. Large circles represent particular populations of neurons; neurons that reside in the cerebellum are enclosed by the dashed box. Vestibular input is conveyed by vestibular afferents that travel in the eighth cranial nerve (n. VIII) and by second-order neurons located in the vestibular nucleus $(V N)$. Mossy fiber inputs also bring eye velocity and retinal slip signals to Purkinje cells. Neurons in the inferior olive provide a potential instructive signal (thick arrows) to Purkinje cells $(P C)$ in the floccular lobe and to floccular target neurons $(F T N)$ located in the medial vestibular nuclcus. The Purkinje cells themselves also carry a potential instructive signal to FT neurons. Purkinje cells inhibit (minus sign) FT neurons. All other connections are excitatory.

the retinal slip signal occurs in one or both of the pathways that converge on the FT neurons.

As shown in Figure $5 B$, floccular Purkinje cells and FT neurons receive the vestibular and retinal slip signals necessary for each of them to be sites of supervised learning (Ito, 1982, 1984; Lisberger, 1988). The instructive signal to both Purkinje cells and to FT neurons could be carried by the inferior olivary neurons. The instructive signal to FT neurons could also be conveyed by the activity of the Purkinje cells themselves, since they are driven by the retinal slip signal. In either case, this activity represents an error signal (Fig. 2) that decreases as the correct VOR gain is acquired.

A neurophysiological correlate of changes in VOR gain is observed in the response properties of floccular Purkinje cells (Miles et al., 1980; Watanabe, 1984). The average responses of these Purkinje cells to a standard rotation of the head are depressed relative to normal in animals with an experimentally induced increase in VOR gain, and are increased in animals with a decreased VOR gain (Lisberger, 1988). The direction of these effects is appropriate, because Purkinje cells provide a tonic inhibitory input to FT neurons (Fig. 5B).

As mentioned in the description of classical conditioning, there is evidence of the capacity of granule cell synapses to undergo anti-Hebbian LTD in culture and slice preparations (Hirano and Hagiwara, 1988; Ito, 1989). VOR gain control could be explained, at least in part, by this learning mechanism operating at synapses between granule cells and Purkinje cells in the floccular lobe. If VOR gain is too low, for example, retinal slip could evoke an increase in climbing fiber input to Purkinje cells that, through anti-Hebbian LTD, would cause a weakening of the highly active vestibular inputs that are signaling head rotation. The effectiveness of these vestibular inputs in driving Purkinje cells would decrease, Purkinje cell responses to head rotation would decrease, and Purkinje cell inhibition on FT neurons would decrease. As a result, the responses of FT neurons to head rotations would increase until the gain of the VOR network is correct and retinal slip is eliminated.

FT neurons also exhibit a neurophysiological correlate of VOR gain adjustment (Lisberger and Pavelko, 1988). The changes in the responsiveness of FT neurons, which parallel changes in VOR gain, could simply reflect synaptic modifications occurring in the cortex, as described above. However, it is equally possible (and is predicted by a recent model of the VOR network; Lisberger and Sejnowski, 1992) that VOR gain control depends on modifications at the synapses between vestibular inputs and FT neurons (Fig. 5B). As mentioned above, the signals necessary for supervised learning converge on FT neurons. If the FT neurons are a site of plasticity and the error signal is carried by the inferior olivary input, the modifications could be accounted for by the mechanisms of Hebbian LTP and LTD. For example, if VOR gain is too low, retinal slip could evoke an increase in inferior olivary input that would potentiate concurrently active vestibular inputs and increase VOR gain. Conversely, if VOR gain is too high, retinal slip in the opposite direction would cause the activity carried by the same inferior olivary inputs to decrease, which, in turn, would trigger the mechanisms of LTD, weakening coactive vestibular inputs and thereby decreasing the gain of the VOR.

Critical aspects of supervised learning in VOR gain control remain to be resolved. One important issue is the degree to which models of the VOR network accurately describe all of the essential components involved in the learning process; the 
A

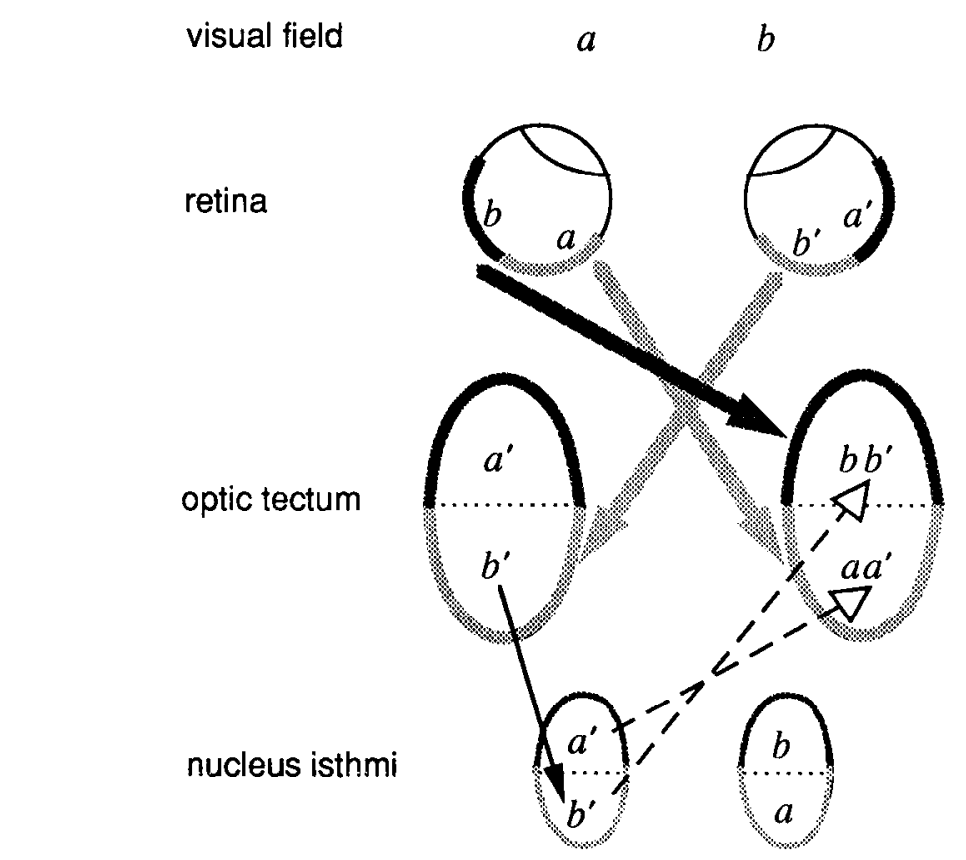

Normal

\section{B Eye Rotated}

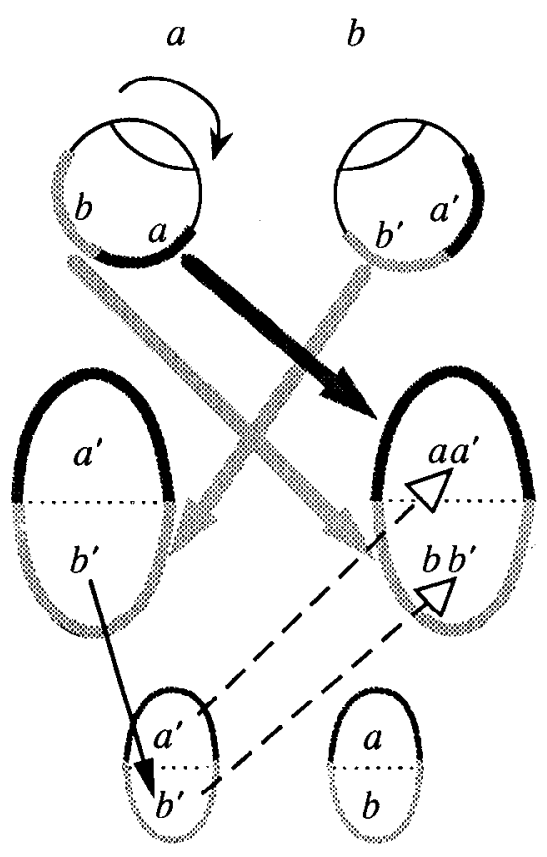

Figure 6. The effect of eye rotation on the axonal projection pattern that underlies binocular visual receptive fields in the right optic tectum of Xenopus. The caudal retina in each eye and the brain regions to which it projects are darkly shaded. The rostral retina in each eye and the regions to which it projects are lightly shaded. I.ocations in the visual field and the sites of their projection onto the left eye retina are indicated as $a$ and $b$. The sites of projection of these locations onto the right eye retina are indicated as $a^{\prime}$ and $b^{\prime}$, respectively. $A$, The normal connection pattern. $B$, The connection pattern that develops following a $180^{\circ}$ rotation of the left eye in a tadpole. Direct retinotectal connections (thick arrows), which are determined by experience-independent mechanisms, instruct the pattern of the isthmotectal conncctions (dashed arrows) so that neurons at a given site in the tectum are activated by the same stimulus in the visual field regardless of which eye provides the visual input.

proposed sites of plasticity have been inferred based on the respective working models that have been employed. More essential is direct evidence of synaptic modifications that correlate with VOR gain adjustment.

\section{Midbrain networks involved in gaze control}

The preceding examples illustrate how supervised learning can select and regulate motor networks. The following examples demonstrate how supervised learning can dictate the transformation and representation of information in sensory networks. Both examples are provided by midbrain networks that control gaze and attention. The goal of these networks is to direct the animal's line of sight toward interesting stimuli in the environment. The essential parametcr of information that is derived and represented by contributing cognitive and sensory (such as auditory, visual, and somatosensory) networks is "locations deserving attention." Spatial information originating from different networks must be transformed into a common format, a task for which supervised learning is particularly well suited. In addition, in order to bring the animal's gaze accurately to a desired location, the derived spatial information must be interpreted and acted upon precisely by the motor network, a task for which supervised learning is required as well. Thus, this system is a microcosm of many of the sensory, associational, and motor processes that are performed by the brain in general, but one in which the essential parameter of information (location) is clear.

A nodal point in the gaze control system is the optic tectum, a structure also referred to as the superior colliculus in mam- mals. Neural activity from various sensory modalities and cognitive centers converges in the optic tectum, bringing to it information about the locations of stimuli (Stein and Meredith, 1993). The spatial information is encoded as foci of activity in a topographic, multimodal map of space. When an animal decides to orient its gaze toward a particular stimulus, output from the corresponding portion of the tectum activates areas in the brainstem tegmentum that translate the topographic code for space into appropriate motor control signals (Masino and Knudsen, 1990; Sparks and Mays, 1990). The degree to which spatial information from each sensory modality is successfully transformed into the common representation can be assessed quantitatively in the optic tectum from the mutual alignment of unit reccptive ficlds measured for the different sensory modalities.

Development of binocular receptive fields. In the frog Xenopus, the portion of the visual field that is viewed by both eyes is represented in the optic tectum by a single visual map, on which the projections from both eyes are congruent. This congruent alignment of visual space maps is achieved by supervised learning (Gaze et al., 1970; Udin, 1985).

The projection from the retina to the contralateral tectum (thick arrows in Fig. $6 \mathrm{~A}$ ) is established early in the development of a Xenopus tadpole, oriented and refined by mechanisms that operate independently of visual experience (Gaze et al., 1979; Keating et al., 1986). Weeks later, during metamorphosis from tadpole to frog, an indirect retinotectal projection arrives at the ipsilateral tectum (Beazley et al., 1972; Grant and Keating, 1986). This pathway originates in the ipsilateral eye, travels via the contralateral optic tectum to the nucleus isthmi, and from there 
NORMAL

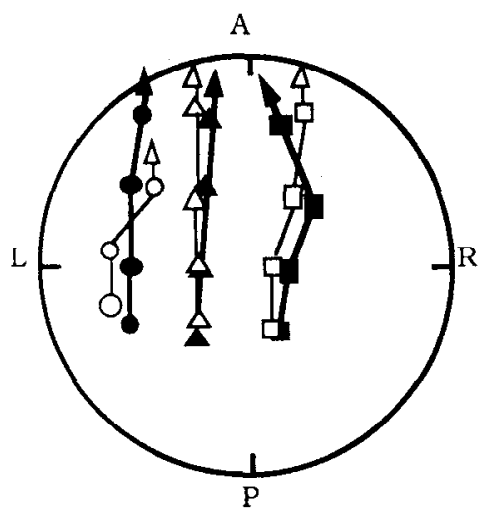

ROTATED AS TADPOLE

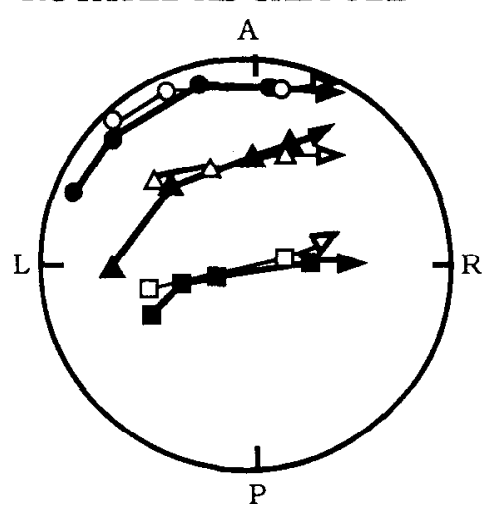

TECTUM

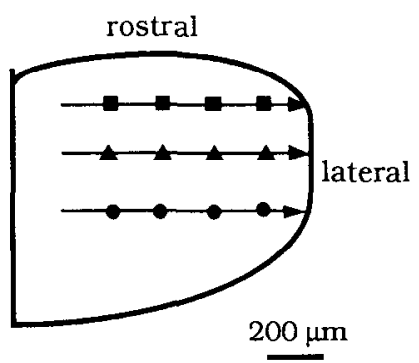

Figure 7. Effect of premetamorphic eye rotation on the relative alignment of left-eye and right-eye receptive fields in the optic tectum of postmetamorphic Xenopus. The large circles represent the visual field (above the animal). Each symbol indicates the center of a visual receptive field from the left eye (contralateral; solid symbols) or right eye (ipsilateral; open symbols), as determined by the region of space in which a visual stimulus produced a strong unit response. The data on the left were gathered from a normal frog. The data in the middle were gathered from a frog whose left eye cup was rotated clockwise about $90^{\circ}$ as a tadpole. On the right is a dorsal view of the right optic tectum showing the locations at which the recordings were made. $A$, anterior visual field; $P$, posterior visual field; $L$, left visual field; $R$, right visual field. Data from Scherer and Udin (1989).

to the ipsilateral optic tectum (Fig. 6A). It is initially oriented by mechanisms that operatc independently of visual experience (Keating and Kennard, 1987). Isthmotectal axons transmitting activity that originates from the caudal portion of the retina project broadly to the posterior portion of the ipsilateral tectum, and axons transmitting activity from the rostral portions of the retina project broadly to the rostral portion of the tectum (Fig. $6 A$ ). The refinement and ultimate topography of this projection, however, are controlled by mechanisms of supervised learning: visual input from the contralateral eye instructs the pattern of connections from the ipsilateral eye that arrive via the nucleus isthmi (Gaze et al., 1970; Udin, 1985).

The contribution of supervised learning to this process of binocular map alignment can be appreciated by considering the effects on the visual space map of surgical rotation of the left eye in a tadpole (Udin and Keating, 1981; Fig. 6B). Because the retinotectal projection from the left eye to the contralateral tectum is oriented independently of visual experience, the visual space map (represented by points $a$ and $b$ in Fig. $6 B$ ) in the right tectum will be rotated relative to normal. During later development, this rotated visual space map acts as an activity template (Fig. 2), altering the projection from nucleus isthmi and causing the map from the right eye (represented by $a^{\prime}$ and $b^{\prime}$ in Fig. $6 B$ ) to be rotated in a concordant fashion. Thus, as a result of rotating the left eye, the right optic tecturn develops a rotated visual space map in which the maps projected from both eyes are nevertheless aligned (Fig. 7).

Changes in the topography of the ipsilateral visual map are due to changes in the anatomy of the isthmotectal projection (Udin and Keating, 1981; Udin, 1989). This is the first and, so far, the only example in a vertebrate nervous system in which supervised learning has been shown to result in anatomical reorganization. Retrograde tracers injected into the tectum of frogs raised with one eye rotated show that the topographic pattern of anatomical projection from the nucleus isthmi to the tectum is altered correspondingly. The mechanisms that disengage isthmotectal axons from sites where their visual receptive fields do not align with those from the contralateral eye and cause them to grow to sites where their visual receptive field do align with those from the contralateral eye have not been determined.
However, the mechanisms underlying Hebbian LTP and LTD are suspected. Activation of NMDA receptors has been shown to induce Hebbian LTP in the optic tecta of distantly related mammals (Miyamoto et al., 1990) and fish (Schmidt, 1990), and, in Xenopus, activation of NMDA receptors is required for the anatomical shift in the isthmotectal projection to occur (Scherer and Udin, 1989). One possibility, therefore, is the following. At an early stage in development, input connections from the contralateral eye become dominant while projections from the nucleus isthmi remain relatively diffuse and modifiable. At this stage, synapses of axon collaterals driven by the ipsilateral eye whose activity is not correlated with that of the instructive input are weakened by the mechanisms of Hebbian LTD, and subsequently these axon collaterals retract. Because the isthmotectal axons lack strong synaptic connections with target neurons, they continue to grow making a series of tentative connections with neurons throughout the tectum. When an axon finally encounters a tectal ncuron whose visually driven activity is correlated with its own, its connection is strengthened by the mechanisms of LTP and stabilized (Scherer and Udin, 1989).

Thus, a fundamental aspect of the process of binocular receptive field alignment in the optic tectum was revealed by the experimental intervention of rotating one eye: in normal development, the pattern of connectivity that aligns the information brought to the tectum from the contralateral and ipsilateral eyes is controlled by supervised learning mediated by activity from the contralateral eye.

Calibration of the auditory space map. Supervised learning also dictates the topography of the auditory space map in the optic tectum. In this example, the brain uses referent information from one network (visual system) to instruct a precise and complex remapping of information from another network (auditory system). Auditory space is a computed parameter, since the spectral content but not the location of a sound source is represented topographically in the cochlea. Source location is derived by the central auditory system by evaluating a set of cues (Fig. 8). The most important of these are frequency-specific, interarual differences in timing (ITD) and level (ILD) of the auditory signal, resulting from the interaction of the head and external ears with the sound field. ITDs reflect the distance 
between the ears (i.e., the size of the head). ILDs result primarily from acoustic shadowing and collecting effects of the head and pinnae. Differences in the sensitivities of the two ears, which can occur normally and which typically accompany conductive hearing impairments, also add to ILDs conveyed to the brain. To interpret sound localization cues correctly, the brain must learn how the auditory periphery shapes acoustic stimuli and, specifically, how values of ITD and ILD correspond with locations in space.

Associations of auditory cue values with locations in space are guided by supervised learning (Held, 1955; Kalil and Freedman, 1967; Knudsen, 1984). The dominant instructive signal is provided by vision (Knudsen and Knudsen, 1989), which is a much more reliable source of spatial information than hearing. Whenever there is a discrepancy between auditory and visual localization of a stimulus, the brain adjusts auditory localization to match visual localization (which is the basis for ventriloquism; Shelton and Searle, 1980; Warren et al., 1982). This principle also applies to the localization of auditory stimuli for the control of gaze, as has been shown for young barn owls made to experience a prismatically displaced visual world (Knudsen and Knudsen, 1990). They gradually adjust their orienting responses to acoustic stimuli according to the optical displacement imposed by the prisms.

A neural correlate of this visually based calibration of auditory gaze control is found in the mutual alignment of auditory and visual unit receptive fields in the owl's optic tectum (Knudsen, 1982). Multimodal units in the optic tectum are tuned for sound source location, and normally their auditory receptive fields align with their visual receptive fields. But after fitting young owls with displacing prisms, the projection of the visual world onto the retinae is shifted. This causes a shift in the representation of the visual world in the optic tectum, as well as a shift in the Iocations of visual receptive fields relative to auditory receptive fields for tectal units (Fig. 9A). After the owls experience a displaced visual field for a period of weeks, the locations of auditory receptive fields in the tectum change (Fig. 9), bringing them into concordance with the optically displaced visual receptive fields (Knudsen and Brainard, 1991). The resultant changes in the auditory space map involve a change in the region of space that is magnified by the map, from the normal overrepresentation of the region directly in front of the animal to an overrepresentation of the region of space corresponding to the optically displaced line of sight, and a change in the region of space that is represented near the edges of the map.

An apparently similar result can be observed in newborn ferrets with one surgically deviated eye (King et al., 1988). Auditory receptive fields in the optic tectum shift in the direction that corresponds with the deviation of the eye. In this case, however, it is not clear whether the change in auditory spatial tuning results from visually guided supervised learning or from altered eye motor control signals, which also can cause systematic changes in the locations of auditory receptive fields (Jay and Sparks, 1984).

Supervised learning responsible for the prism-induced shift of the auditory space map in the owl's optic tectum occurs in the external nucleus of the inferior colliculus (ICX), a primary source of auditory input to the optic tectum (Knudsen and Knudsen, 1983). In the ICX, auditory localization cues are combined across frequency channels to create a topographic representation of auditory space (Fig. 8). In prism-reared owls, the space map in the ICX is shifted in the direction and by an amount

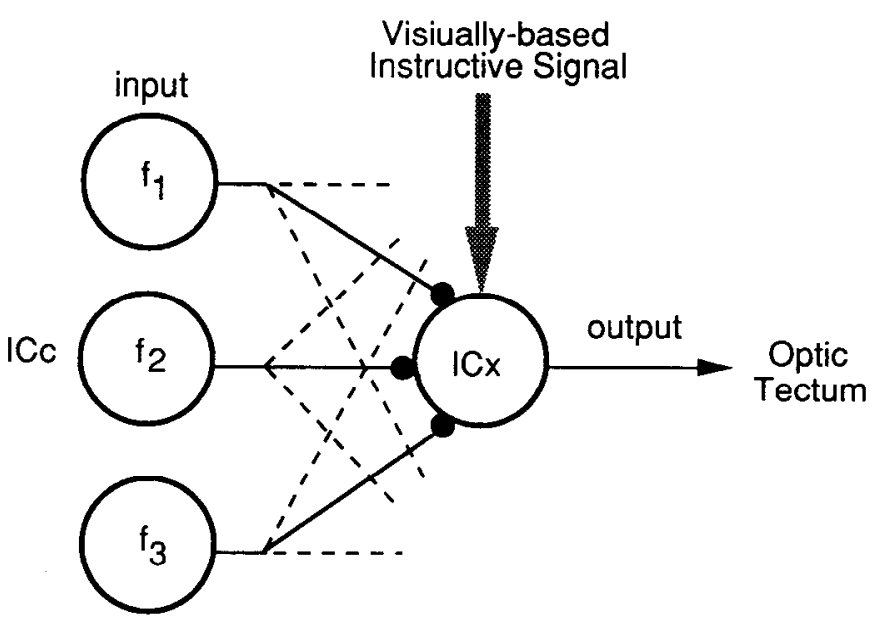

frequency specific auditory cues

auditory space

Figure 8. The auditory network that combines information across frequency channels to create spatial receptive fields in the ICx of the barn owl. Large circles represent populations of neurons in the central nucleus $(I C C)$ and external nucleus $(I C X)$ of the inferior colliculus. Each neuron in the ICc responds selectively to specific values of sound localization cues within a narrow band of frequency $\left(f_{i}, f_{2}\right.$, or $\left.f_{3}\right)$. Small solid circles represent strong connections between ICc neurons and neurons in the ICX that encode locations in space. Dashed lines represent weakened connections of ICC neurons with other ICX neurons (not shown) encoding different locations in space. These connections have been weakened (or eliminated) by the learning process. The cue values that excite an IC $x$ neuron, and hence the location of its spatial receptive field, are determined by a visually based instructive signal (thick arrow) of unknown origin.

that completely accounts for the shift of the map in the optic tectum (Brainard and Knudsen, 1993). In contrast, the representation of localization cue values in the central nucleus of the inferior colliculus ( $\mathrm{ICc}$ ), which immediately precedes the ICx (Fig. 8), is normal in prism-reared owls with shifted space maps in the ICx (Brainard and Knudsen, 1993). This indicates that the visually based instruction of auditory receptive field locations takes place at the level of the ICx, the site where the auditory space map is synthesized from frequency-specific cues.

Neither the identity of the neurons that carry the instructive signal, nor even the nature of the instructive signal, is known in this system. One hypothesis is that a retinotopic visual map provides an activity template (Fig. 2) for the auditory map in the ICx: activation of a site in the ICx by a visual instructive signal in response to a bimodal (auditory-visual) stimulus could result in the strengthening of all concurrently active auditory inputs at that site. However, no anatomical or physiological evidence has been found in the owl of direct or indirect visual input to the ICX. A second hypothesis is that another part of the brain (perhaps the tectum) compares auditory and visual spatial information and transmits the result in the form of an error signal (Fig. 2) to the ICx that modifies the connectivity in the ICx. A third hypothesis is that the instructive signal results from visual evaluation of the accuracy of auditory orientating responses to bimodal stimuli. According to this hypothesis, an error signal is generated based on the discrepancy between the direction of gaze resulting from an auditory orientating response and the location of the stimulus as indicated by vision. This model requires that an electrical or biochemical trace of the auditory activity pattern that led to stimulus localization persists 
A
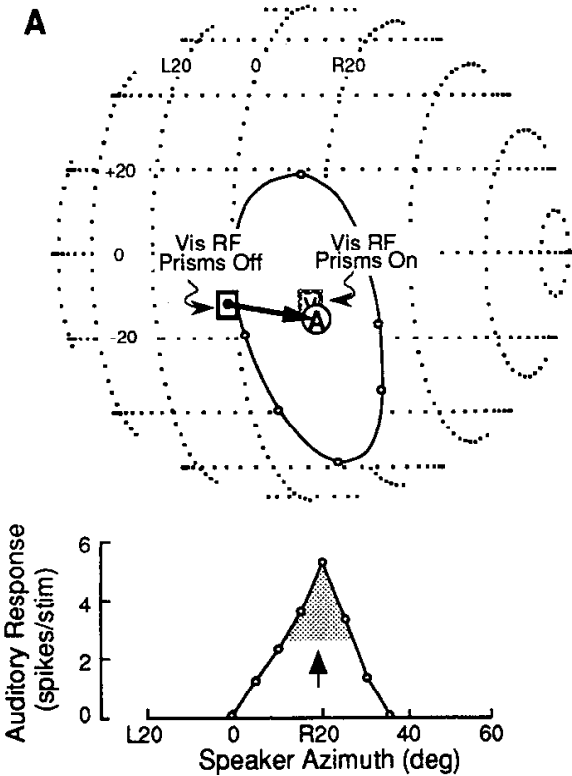

Auditory Response (spikes/stim)
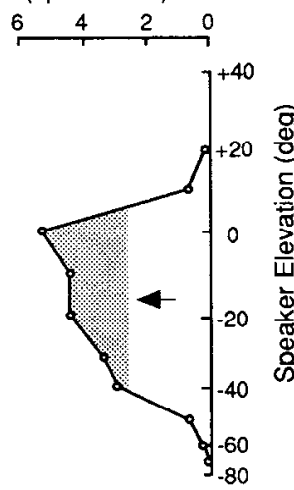

B

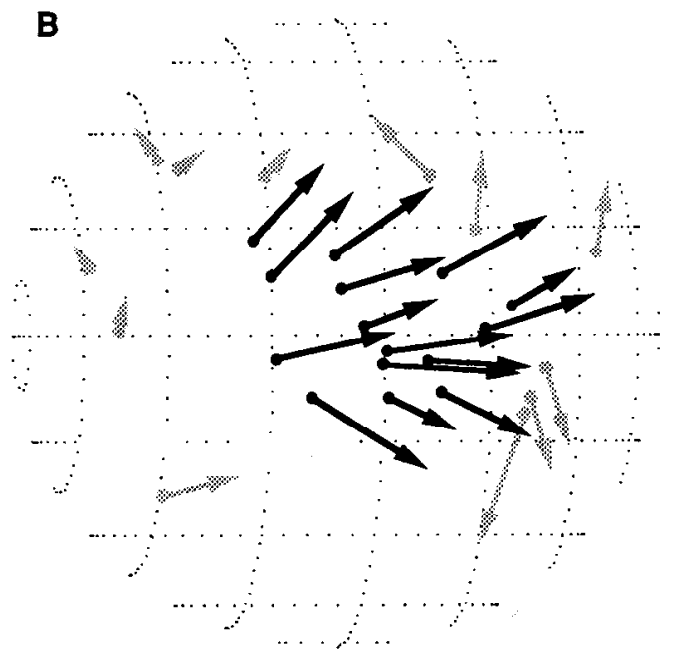

Figure 9. The effect of prism rearing on the alignment of auditory and visual receptive fields in the optic tectum of the barn owl. These electrophysiological data were recorded from single units in an owl raised with optical prisms that displaced the frontal portion of the visual field $23^{\circ}$ to the left, corresponding to a $23^{\circ}$ rightward displacement of unit visual receptive fields. Because the eyes of owls are essentially stationary in the head, the optic displacement caused by the prisms cannot be compensated for by adjustments in eye position, as it is in humans. In $A$, the visual and auditory receptive fields of a single unit are plotted on a globe of space. The locations of the visual receptive field (Vis $R F$ ) with the prisms on and off are represented by boxes. The auditory receptive field is the vertical elliptical area. The circled $A$ indicates the center of the auditory best area, the region of space in which a test sound produced more than $50 \%$ of the maximum response. The auditory best area was derived from responses to 10 repetitions of a $100 \mathrm{msec}$ broad-band noise burst, as shown below and to the right of the globe; the arrows indicate the best area center. The vector on the globe indicates the direction and magnitude of the misalignment between the center of the visual receptive field with the prisms off and the center of the auditory best area. This misalignment matches the optical displacement of the unit's visual receptive field. In $B$, vectors represent the misalignments between visual receptive field centers (vector origins) and auditory best area centers (vector heads) for numerous recording sites across the optic tectum of the same owl measured with the prisms off. Normally, visual receptive fields and auditory best areas are mutually aligned and the vectors are short and oriented randomly. In this owl, auditory best areas were shifted in the tectum, whereas visual receptive fields were located normally. Auditory best areas were shifted systematically only for units with visual receptive fields located in the frontal portion of the visual field that was displaced by the prisms (solid vectors). Units representing the more peripheral portions of the visual field (shaded vectors), which were blocked or unaffected by the prisms, did not exhibit systematic shifts in auditory best areas. As a result, large discontinuities in the auditory space map occurred at the representation of the boundary between these zones of the visual field. Data from Knudsen and Brainard (1991).

in the ICx until the visually based error signal arrives to shape it.

Although the instructive mechanism remains obscure in this system, the visual modification of an auditory space map illustrates the capacity of supervised learning to dictate the topography of brain maps. There are numerous examples of experience-dependent changes in brain maps at various levels in the auditory, visual, and somatosensory pathways (Devor and Wall, 1978; Jenkins et al., 1990; Kaas, 1991; Pons et al., 1991; Gilbert and Wiesel, 1992; Recanzone et al., 1993). Virtually all of these examples, however, reflect primarily the effects of unsupervised learning, by which the brain continuously modulates map topography on the basis of activity-dependent competition between the afferents that convey the mapped information. When supervised learning occurs, however, adjustments in map topography due to competition between afferents are overwhelmed by the shaping influence of the instructive signal. In the case of owls raised with displacing prisms, for example, supervised learning changes the region of auditory space that is represented in the map, expands the representation of locations corresponding to the optically displaced line of sight, and shrinks the representation of locations corresponding to the normal line of sight (Knudsen and Brainard, 1991; Knudsen and Brainard, 1991). In addition, sharp discontinuities are created in the map at sites that represent the edges of the prisms, corresponding to the transition from a displaced to a normal visual field (Fig. $9 B$ ). In owls that are deprived of the instructive influence of vision through binocular lid closure, the auditory map does not acquire normal topography and the orientation of the map can flip upside-down (Knudsen et al., 1991). In dark-reared guinea pigs, no auditory space map forms at all (Withington-Wray et al., 1990). Visually based, supervised learning controls these aspects of auditory map topography, despite the fact that the afferent activity that conveys auditory spatial information to the map is itself unaltered.

\section{Concluding remarks}

Supervised and unsupervised forms of learning cooperate during development, and in adulthood, to optimize the integrative mechanisms of the brain for the individual. Early in the development of a network, basic patterns of connectivity form by the interaction of genetic determinants and unsupervised forms of learning (Miller, 1989; Brown et al., 1991). This ability of networks to self-organize allows the brain to develop extensively before an animal is even born. As the brain begins to process experience-driven activity, however, supervised learning begins to exert its effects, adjusting and calibrating the original patterns of connectivity. In adulthood, supervised and unsupervised forms 
of learning continue to adjust connectivity patterns in many networks, altering them adaptively in response to changes in sensory or motor capacities and/or environment that may occur throughout the lifetime of the animal.

The powerful influence that supervised learning can exert on patterns of connectivity in the brain is shown by many examples. In the case of classical conditioning, strong connections are established between networks of neurons that are not functionally coupled prior to conditioning (Pavlov, 1927; McCormick and Thompson, 1984; Thompson, 1990). In the development of binocular receptive fields in the optic tectum of Xenopus, the pattern of activity from the contralateral eye instructs the anatomical pattern of tectal connections representing input from the ipsilateral eye (Udin and Kcating, 1981; Udin, 1989). In the cast of the auditory space map in the owl, connections between pre- and postsynaptic neurons are strengthened if the information conveyed by the auditory inputs is consistent with the visual instructive signal, and are weakened if the information conveyed by the inputs is inconsistent with the instructive signal (Brainard and Knudsen, 1993). Thus, every aspect of the auditory space map, including its orientation, position, and topography, is under the control of supervised learning.

The adjustments made by supervised learning in the patterns of connectivity are precise and customized for the individual. The gain of the VOR in the monkey is maintained near a value of 1 , despite differences in the sensitivity of the vestibular end organs or the strength of the extraocular muscles that may occur among individuals and within an individual as it ages (Lisberger, 1988). Auditory units in the barn owl's tectal space map are tuned to values of ITD and ILD that are within tens of microscconds and a few decibcls of the values that correspond with their visual receptive fields, despite individual variability in these correspondences (Olsen et al., 1989; Knudsen et al., 1991). Binocular neurons in the Xenopus tectum maintain aligned receptive ficlds from the left and right eyes. despite changes in the positions and orientations of the eyes during development (Gaze et al., 1970: Keating and Kennard. 1987). Thus, supervised learning adapts network connectivity patterns to the world of the individual with a precision that could not develop autonomously under the sole control of the genome.

The instructional signal for some networks is an activity template provided by another network (Fig. 2). For networks that opcrate on diverse sets of inputs. an activity template can organize the representation of those various inputs according to the coordinate space dictated by the template. For example, in sensory systems, this would enable independent information about a stimulus feature that is processed in different networks to be mutually calibrated and combined into a common representation of that feature. In motor systems, this would enable cognitive and sensory inputs to be organized into a coordinate space that is based on movements or muscle pull-directions. When the referent pattern conveys a specific meaning, such as might originate from networks involved in recognition, for example, supervised learning could confer that meaning on the output activity pattern of the network.

It is not clear how much of the brain's functional organization is controlled by supervised learning, because the capacities of supervised and unsupervised learning to shape connectivity patterns overlap extensively. Mechanisms that can account for the known examples of supervised learning. that is. those that underlic LTP and LTD and anti-Hebbian LTD, can also account for instances of unsupcrvised learning. A simplifying hypothesis is, therefore, that the same set of mechanisms operates to implement both forms of learning. The difference could be that in unsupervised learning all inputs are equally modifiable, whereas in supervised learning the inputs that provide the instructive signal maintain a dominating influence on postsynaptic neurons.

The dominance of an instructive input could be mediated by strong synapses that stabilize first in development or are stabilized preferentially by the postsynaptic neuron. Such stable synapses may exhibit a distinguishing anatomical or histochemical phenotype. It will be important to characterize the anatomical and biochemical properties of synapses made by known instructive neurons, since a distinguishing synaptic phenotype would facilitate greatly the identification of brain regions whose functional organization is controlled by supervised learning, as well as the identification of brain regions that are the source of such instructive signals.

It is of course, premature to presume that all instances of supervised learning depend on the mechanisms of Hebbian LTP and LTD or anti-Hebbian LTD operating in combination with a dominating instructional input. Although circumstantial evidence is accumulating for the role of these mechanisms in the biological networks discussed here, in none of them has the cellular mechanisms of learning actually been identified. Moreover, there is a class of supervised networks, such as sensorimotor systems controlling goal-directed movements, in which network computations are completed and information is transmitted long before an error signal is available to shape the network's connectivity. For adjustments to occur under these conditions, some electrophysiological or biochemical trace of the computation must persist in the network until the error signal arrives to shape its connectivity. Neither the rules nor the mechanisms by which such instruction is implemented are known. Thus, research on these as well as on other instances of supervised learning may elucidate entirely new classes of rules and mechanisms of learning in the brain.

\section{References}

Anderson JA. Rosenfeld E (1988) Neurocomputing: foundations of research. Cambridge, MA: MIT Press.

Anderson JA, Rosenfeld E, Pellionisz A (1990) Neurocomputing If: directions of research. Cambridge, MA: MIT Press.

Balaban CD (1984) Olivo-vestibular and cercbello-vestibular connections in albino rabbits. Ncuroscience 12:120-149.

Beazley L. Keating MJ. Gaze RM (1972) The appearance during development of responses in the optic tectum following visual stimulation of the ipsilateral cye in X'enopus laevis. Vision Res 12:407-410.

Bindman LJ, Murphy KPSJ, Pockett S (1988) Postsynaptic control of the induction of long-term changes in efficacy of transmission at neocortical synapses in slices of rat brain. J Neurophysiol 60:1053-1065.

Black AH. Prokasy WF. eds (1972) Classical conditioning II: current research and theory. New York: Appleton-Century-Crofts.

Bliss TVP. Lomo T (1973) Long-lasting potentiation of synapse transmission in the dentate area of the anesthetized rabbit following stimulation of the perforant path. J Physiol (Lond) 232:331-356

Brainard MS, Knudsen El (1993) Experience-dependent plasticity in the inferior colliculus: a site for visual calibration of the neural representation of auditory space in the barn owl. J Neurosci 13:45894608 .

Brown TH. Chapman PFE. Kairiss W, Keenan CL (1988) Long-term synaptic potentiation. Science 242:724-728

Brown TH, Zador AM, Mainen ZF, Claiborne BJ (1991) Hebbian modifications in hippocampal neurons. In: LTP: a debate of current issues (Baudry M, Davis J, eds), pp 357-389. Cambridge, MA: MIT Press.

Calabresi P. Maj R. Pisani A, Mercuri NB. Bernardi G (1992) Longterm synaptic depression in the striatum: physiological and pharmacological characterization. J Neurosci 12:4224-4233. 
Devor M. Wall PD (1978) Reorganisation of spinal cord sensory map after peripheral nerve injury. Nature 276:75-76.

Gaze RM, Keating MJ, Székely G, Beazley L (1970) Binocular interaction in the formation of specific intertectal neuronal connexions. Proc R Soc Lond [Biol] 175:107-147.

Gaze RM, Keating MJ, Östberg A, Chung S-H (1979) The relationship between retinal and tectal growth in larval Xenopus: implications for the development of the retino-tectal projection. J Embryol Exp Morphol 53:103-143.

Georgopoulos AP (1986) On reaching. Annu Rev Neurosci 9:147170.

Gilbert CD, Wiesel TN (1992) Receptive field dynamics in adult primary visual cortex. Nature 356:150-152.

Grant S, Keating MJ (1986) Normal maturation involves systematic changes in binocular visual connections in Xenopus laevis. Nature $322: 258-261$.

Hebb D (1949) The organization of behavior. New York: Wiley.

Held R (1955) Shifts in binaural localization after prolonged exposures to atypical combinations of stimuli. Am J Psychol 68:526-548.

Hirano T, Hagiwara S (1988) Synaptic transmission between rat cerebellar granule and Purkinje cells in dissociated cell culture: effects of excitatory-amino acid transmitter agonists. Proc Natl Acad Sci USA 85:934-938.

Ito M (1982) Cerebellar control of the vestibulo-ocular reflex - around the flocculus hypothesis. Annu Rev Neurosci 5:725-796.

Ito M (1984) The cerebellum and neural control. New York: Appleton Century-Crofts.

Ito M (1989) Long-term depression. Annu Rev Neurosci 12:85-102.

Jay MF, Sparks DL (1984) Auditory receptive fields in primate superior colliculus shift with changes in eye position. Nature 309:345347.

Jenkins WM, Merzenich MM, Ochs MT, Allard T, Guic-Robles E (1990) Functional reorganization of primary somatosensory cortex in adult owl monkeys after behaviorally controlled tactile stimulation. J Neurophysiol 63:82-104.

Kaas JH (1991) Plasticity of sensory and motor maps in adult mammals. Annu Rev Neurosci 14:137-167.

Kalil R, Freedman SJ (1967) Compensation for auditory rc-arrangcment in the absence of observer movement. Percept Motor Skills 24: $475-478$.

Keating MJ, Kennard C (1987) Visual experience and the maturation of the ipsilateral visuotectal projection in Xenopus laevis. Neuroscience 21:519-528.

Keating MJ, Grant S, Dawes EA, Nanchahal K (1986) Visual deprivation and the maturation of the retinotectal projection in Xenopus laevis. J Embryol Exp Morphol 91:101-115.

King AJ, Hutchings ME, Moore DR, Blakemore C (1988) Developmental plasticity in the visual and auditory representations in the mammalian superior colliculus. Nature 332:73-76.

Kirkwood A, Dudek SM, Gold JT, Aizenman CD, Bear MF (1993) Common forms of synaptic plasticity in the hippocampus and neocortex in vitro. Science 260:1518-1521.

Knudsen EI (1982) Auditory and visual maps of space in the optic tectum of the owl. J Neurosci 2:1177-1194.

Knudsen EI (1984) The role of auditory experience in the development and maintenance of sound localization. Trends Neurosci 7:326-330.

Knudsen EI, Brainard MS (1991) Visual instruction of the neural map of auditory space in the developing optic tectum. Science 253:85-87.

Knudsen EI, Brainard MS (in press) Creating a unified representation of visual and auditory space in the brain. Annu Rev Neurosci, in press.

Knudsen EI, Knudsen PF (1983) Space-mapped auditory projections from the inferior colliculus to the optic tectum in the barn owl (Tyto alba). J Comp Neurol 218:187-196.

Knudsen EI, Knudsen PF (1989) Vision calibrates sound localization in developing barn owls. J Ncurosci 9:3306-3313.

Knudsen EI, Knudsen PF (1990) Sensitive and critical periods for visual calibration of sound localization by barn owls. J Neurosci 10: 222-232.

Knudsen EI, Esterly SD, du Lac S (1991) Stretched and upside-down maps of auditory space in the optic tectum of blind-reared owls; acoustic bias and behavioral correlates. J Neurosci 11:1727-1747.

Krupa DJ, Thompson JK. Thompson RF (1993) Localization of a memory trace in the mammalian brain. Science 260:989-991.

Lisberger SG (1988) The neural basis for learning of simple motor skills. Science $242: 728-735$.
Lisberger SG, Miles FA (1980) Role of primate medial vestibular nucleus in long-term adaptive plasticity of vestibuloocular reflex. $J$ Neurophysiol 43:1725-1745.

Lisberger SG, Pavelko TA (1988) Brain stem neurons in modified pathways for motor learning in the primate vestibulo-ocular reflex. Science 242:771-773

Lisberger SG, Sejnowski TJ (1992) Motor learning in a recurrent network model based on the vestibulo-ocular reflex. Nature 360:159161.

Llinás R, Hillman RE (1969) Physiological and morphological organization of the cerebellar circuits in various vertebrates. In: Neurobiology of cerebellar evolution and development (Llinás R, ed), pp 43-73. Chicago: American Medical Association, Education and Research Foundation

Lomo T (1966) Frequency potentiation of excitatory synaptic activity in the dentate area of the hippocampal formation. Acta Physiol Scand [Suppl] 68:128.

Marr D (1969) A theory of cerebellar cortex. J Physiol (I.ond) 202 $437-470$.

Masino T, Knudsen El (1990) Horizontal and vertical components of head movement are controlled by distinct neural circuits in the barn owl. Nature 345:434-437.

Mauk MD, Steinmetz JE, Thompson RF (1986) Classical conditioning using stimulation of the inferior olive as the unconditioned stimulus. Proc Natl Acad Sci USA 83:5349-5353.

McCormick DA. Thompson RF (1984) Neuronal responses of the rabbit cerebellum during acquisition and performance of a classically conditioned nictitating membrane-eyelid response. J Neurosci 4:28112822

McCormick DA, Steinmetz JE, Thompson RF (1985) Lesions of the inferior olivary complex cause extinction of the classically conditioned eyeblink response. Rrain Res 359:120-130

Miles FA, Braitman DJ (1980) Long-term adaptive changes in primate vestibuloocular reflex. II. Electrophysiological observations on semicircular canal primary afferents. J Neurophysiol 43:1426-1436.

Miles FA, Eighmy BB (1980) Long-term adaptive changes in primate vestibuloocular reflex. I. Behavioral observations. J Neurophysiol 43 1406-1424.

Miles FA, Braitman DJ, Dow BM (1980) Long-term adaptive changes in primate vesitbuloocular reflex. IV. Electrophysiological observations in flocculus of adapted monkeys. J Neurophysiol 43:147\%-1493.

Miller KD (1989) Correlation-based models of neural development. In: Neuroscience and connectionist theory (Gluck MA, Rumelhart DE, eds), pp 267-352. Hillsdale, NJ: Erlbaum.

Miyamoto T, Sakurai T, Okada Y (1990) Masking effect of NMDA receptor antagonists on the formation of long-term potentiation (LTP) in superior colliculus slices from the guinea pig. Brain Res 518:166172

Mulkey RM, Malenka RC (1992) Mechanisms underlying induction of homosynaptic long-term depression in area CAl of the hippocampus. Neuron 9:967-975.

Olsen JF, Knudsen EI, Esterly SD (1989) Neural maps of interaural time and intensity differences in the optic tectum of the barn owl. J Neurosci 9:2591-2605

Pavlov IP (1927) Conditioned reflexes: an investigation of the physiological activity of the cerebral cortex (Anrep GV, trans). London: Oxford UP.

Pons TP, Garraghty PE, Ommaya AK, Kaas JH, Taub E, Mishkin M (1991) Massive cortical reorganization after sensory deafferentation in adult macaques. Science 252:1857-1860.

Recanzone GH, Schreiner CE, Merzenich MM (1993) Plasticity in the frequency representation of primary auditory cortex following discrimination training in adult owl monkeys. J Neurosci 13:87-103.

Scherer WJ, Udin SB (1989) $N$-methyl-D-aspartate antagonists prevent interaction of binocular maps in Xenopus tectum. J Neurosci 9:3837-3843

Schmidt JT (1990) Long-term potentiation and activity-dependent retinotopic sharpening in the regenerating retinotectal projection of goldfish: common sensitive period and sensitivity to NMDA blockers. J Neurosci 10:233-246.

Shelton BR, Searle CL (1980) The influence of vision on the absolute identification of sound-source position. Percept Psychophysiol 28: 589-596.

Solomon PR, VanderSchaaf ER, Thompson RF, Weisz DJ (1986) Hippocampus and trace conditioning of the rabbit's classically con- 
ditioned nictitating membrane response. Behav Neurosci 100:729744.

Sparks DL, Mays LE (1990) Signal transformations required for the generation of saccadic eye movements. Annu Rev Neurosci 13:309336.

Stein BE, Meredith MA (1993) The merging of the senses. Cambridge, MA: MIT Press.

Steinmetz JE, Rosen DJ, Chapman PF, Lavond DG, Thompson RF (1986) Classical conditioning of the rabbit eyelid response with a mossy fiber stimulation CS. I: Pontine nuclei and middle cerebellar peduncle stimulation. Behav Neurosci 100:871-880.

Steinmetz JE, Lavond DG, Thompson RF (1989) Classical conditioning in rabbits using pontine nucleus stimulation as a conditioned stimulus and inferior olive stimulation as an unconditioned stimulus. Synapse 3:225-232.

Stent GS (1973) A physiological mechanism for Hebb's postulate of learning. Proc Natl Acad Sci USA 70:997-1001.

Thompson RF (1986) The neurobiology of learning and memory. Science 233:941-947.

Thompson RF (1990) Neural mechanisms of classical conditioning in mammals. Philos Trans $\mathrm{R}$ Soc Lond [Biol] 329:161-170.
Udin SB (1985) The role of visual experience in the formation of binocular projections in frogs. Cell Mol Neurobiol 5:85-102.

Udin SB (1989) The development of the nucleus isthmi in Xenopus. II. Branching patterns of contralaterally projecting isthmotectal axons during maturation of binocular maps. Vis Neurosci 2:153-163.

Udin SB, Keating MJ (1981) Plasticity in a central nervous pathway in Xenopus: anatomical changes in the isthmotectal projection after larval eye rotation. J Comp Neurol 203:575-594.

Warren DH, Welch RB, McCarthy TJ (1982) The role of visualauditory "compellingness" in the ventriloquism effect: implications for transitivity among the spatial senses. Percept Psychophysiol 30: 557-564.

Watanabe E (1984) Neuronal events correlated with long-term adaptation of the horizontal vestibulo-ocular reflex in the primate flocculus. Brain Res 297:169-174.

Withington-Wray DJ, Binns KE, Keating MJ (1990) The maturation of the superior collicular map of auditory space in the guinea pig is disrupted by developmental visual deprivation. Eur J Neurosci 2:682692. 